\title{
Reconstruction of post-burn anterior neck contractures using a butterfly design free anterolateral thigh perforator flap
}

\author{
Alexandre G. Lellouch ${ }^{1,2,3}$, Zhi Yang Ng ${ }^{2,3}$, Victor Pozzo ${ }^{1}$, Tabrez Suffee ${ }^{1}$, Laurent A. Lantieri ${ }^{1}$ \\ ${ }^{1}$ Department of Plastic Reconstructive Surgery, European Georges Pompidou Hospital (AP-HP), Paris Descartes University, Paris, France; \\ ${ }^{2}$ Division of Plastic and Reconstructive Surgery, and ${ }^{3}$ Vascularized Composite Allotransplantation Laboratory, Center for Transplantation \\ Sciences, Massachusetts General Hospital, Harvard Medical School, Boston, MA, USA
}

Anterior neck burns represent a major reconstructive challenge due to severe sequalae including restriction in movement and poor aesthetic outcomes. Common treatment options include skin grafting with/without dermal matrices, and loco-regional and distant free flap transfers with/without prior tissue expansion. Such variation in technique is largely influenced by the extent of burn injury requiring resurfacing. In order to optimize like-for-like reconstruction of the anterior neck, use of wide, thin and long flaps such as the anterolateral thigh (ALT) perforator flap have been reported with promising results. Of note, some patients have a tendency towards severe scar contractures, which may be contributed by the greater extent of inflammation during wound healing. We report our experience at 4 years' followup after secondary reconstruction of severe, anterior neck burn contractures in two patients by harvesting the ALT flap with a butterfly design. This technique provides adequate wound resurfacing of the burned neck and surrounding areas, and provides good neck extensibility by addressing both anterior and lateral aspects of the scar defect simultaneously. Such a flap design reduces tension on wound edges and thus, the risk of contracture recurrence in what remains a particularly challenging type of burn reconstruction.

Keywords Free flap / Neck / Reconstructive surgical procedures / Burns / Contracture

\section{Correspondence:}

Alexandre G. Lellouch

Department of Plastic Reconstructive

Surgery, European Georges Pompidou Hospital (AP-HP), Paris Descartes

University, 20 rue Leblanc 75015

Paris, France

Tel: $+33-698372778$

Fax: $+33-156095320$

E-mail: alellouch@mgh.harvard.edu

Received: April 22, 2019 • Revised: September 19, 2019 • Accepted: November 23, 2019

pISSN: 2234-6163 • elSSN: 2234-6171 • https://doi.org/10.5999/aps.2019.00591 • Arch Plast Surg 2020;47:194-197

\section{INTRODUCTION}

Anterior neck burns represent a particular reconstructive challenge as this body area is under continuous tension due to its wide range of motion [1]. The quality and quantity of skin required for satisfactory reconstruction of this area remains a difficult goal [2], and contractures can limit neck extension, cause persistent pain, headaches and depression, leading to a poor quality of life [3]. Various reconstructive techniques have been described including split-thickness skin grafts (STSGs), local flaps, local flaps combined with STSGs, expanded local flaps, thin pedicled flaps, free flaps, and expanded free flaps [4], but patient outcomes are highly variable. In this study, we developed a butterfly design of the anterolateral thigh (ALT) flap to specifically address the post-resection skin defect. We describe the surgical technique involved to achieve restoration of neck extension in burn reconstruction. 


\section{IDEA}

\section{Butterfly design of ALT flap}

Preoperatively, Doppler ultrasound [5] and computed tomography angiography were performed for evaluation of suitable perforators arising from the lateral circumflex femoral system. Intraoperatively, the patient is positioned supine with the neck in hyperextension. Two teams work simultaneously-one excises the anterior neck scar with respect for the aesthetic unit of the neck and prepares the recipient vessels (external jugular vein, facial and superior thyroid arteries) while the other team designs and harvests the ALT flap.

The ALT is designed in a butterfly shape corresponding to the post-resection defect with previously identified perforators located in the middle of the flap (Fig. 1). The flap is then harvested sub-fascial and anastomosed end-to-end to the superior thyroid artery and external jugular vein. Because of the butterfly shape design of the ALT flap (Fig. 2), the donor site can be closed in V-Y fashion at both proximal and distal ends (Fig. 3).

\section{Cases}

Case 1

An 11-year-old boy from Saudi Arabia presented with severe an-

\section{Fig. 1. Flap design}

Butterfly design of anterolateral thigh flap centered over preoperatively identified perforators using a handheld Doppler.

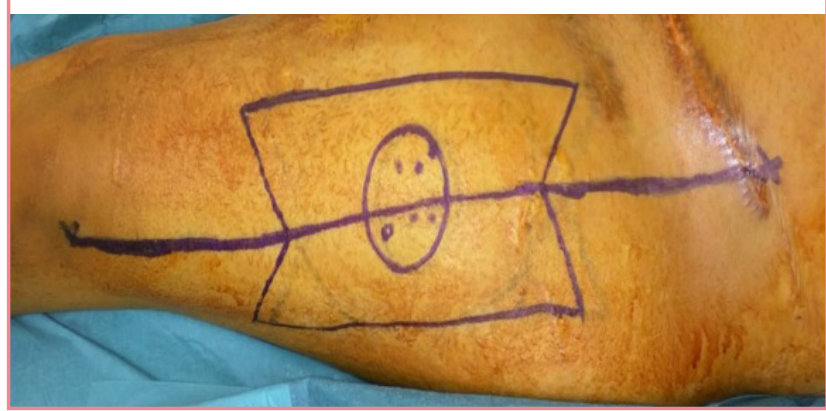

\section{Fig. 2. Intraoperative photograph}

Butterfly design of anterolateral thigh flap after harvest, to match the dimensions of the resection defect of the burned cervical neck.

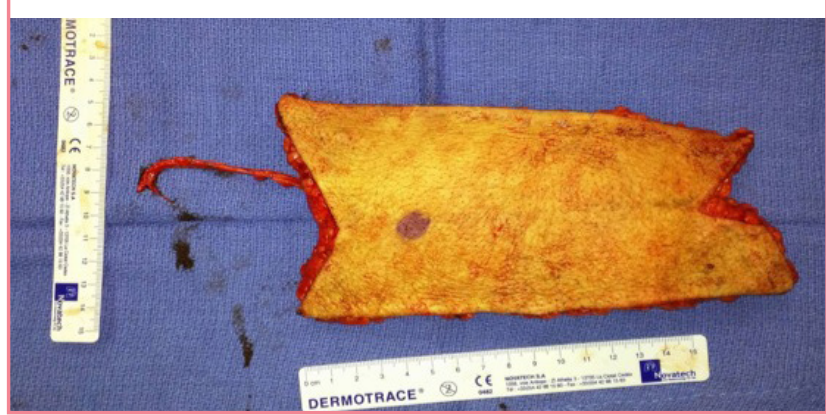

terior cervical contracture (based on range of neck extension according to Makboul's classification system [6]: mild, $95^{\circ}-110^{\circ}$; moderate, $85^{\circ}-95^{\circ}$; severe, $\left.<85^{\circ}\right)$. His parents gave their consent. He had previously sustained thermal burns involving $40 \%$ of total body surface area (TBSA) due to an oven explosion when he was 6 years old, and was treated initially with excision and STSG at another facility. Intraoperatively, the excision defect was $19 \times 10 \mathrm{~cm}$ and resurfaced with a butterfly design ALT flap. At 4 years' follow-up, neck extension had improved from $83^{\circ}$ preoperatively to $120^{\circ}$ without recurrence of scar contracture. Liposuction of the ALT flap was performed at 1-year postoperative to further improve neck contour (Fig. 4).

\section{Case 2}

A 23-year-old woman from Saudi Arabia presented with moderate anterior cervical contracture similar to case 1 . The patient gave her consent. TBSA was $45 \%$ with mainly upper body involvement and she was also initially treated with excision and STSG at 8 years old. The burn scar was excised and resurfaced with a $15 \times 8 \mathrm{~cm}$ butterfly design ALT flap. At 4 years' follow-up, neck extension was $120^{\circ}$ from $90^{\circ}$ preoperatively without any evidence of contracture recurrence. Similarly, she underwent a secondary contouring procedure with liposuction at 1-year postoperative to improve her aesthetic outcome (Fig. 5).

\section{DISCUSSION}

Following burn injury, neck contractures may develop due to various reasons. Most commonly, this results from damage to deeper tissues leading to significant functional and aesthetic deficits of the lower face and neck including the upper chest [7] or progressive contraction and hypertrophic scarring due to failure of skin grafting [8]. When more than two-thirds of the ante-

\section{Fig. 3. Immediate postoperative photograph}

Butterfly design of anterolateral thigh flap allows closure of the donor site in $\mathrm{V}$-Y fashion.

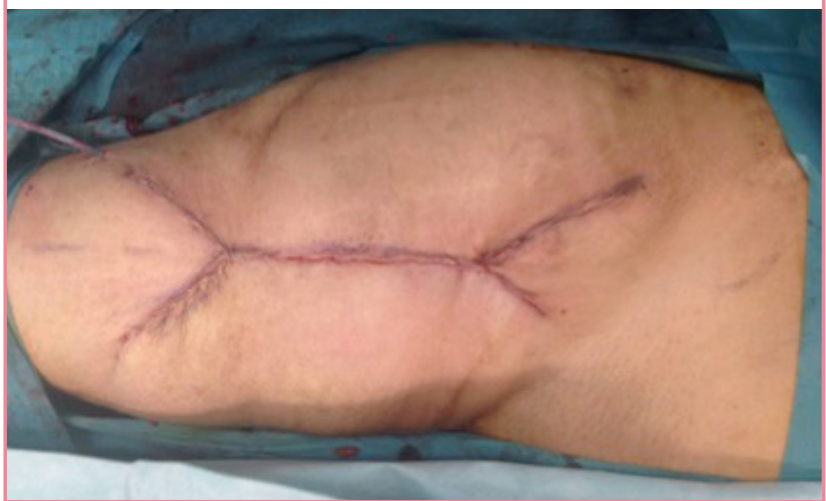




\section{Fig. 4. Case 1}

An 11-year-old boy patient: (A) antero-posterior and (B) lateral views. At 4 years' follow-up following butterfly anterolateral thigh flap resurfacing and additional liposuction (at postoperative 1 year), with acceptable aesthetics and improvement in neck extension (from 83 to $\left.120^{\circ}\right)$.
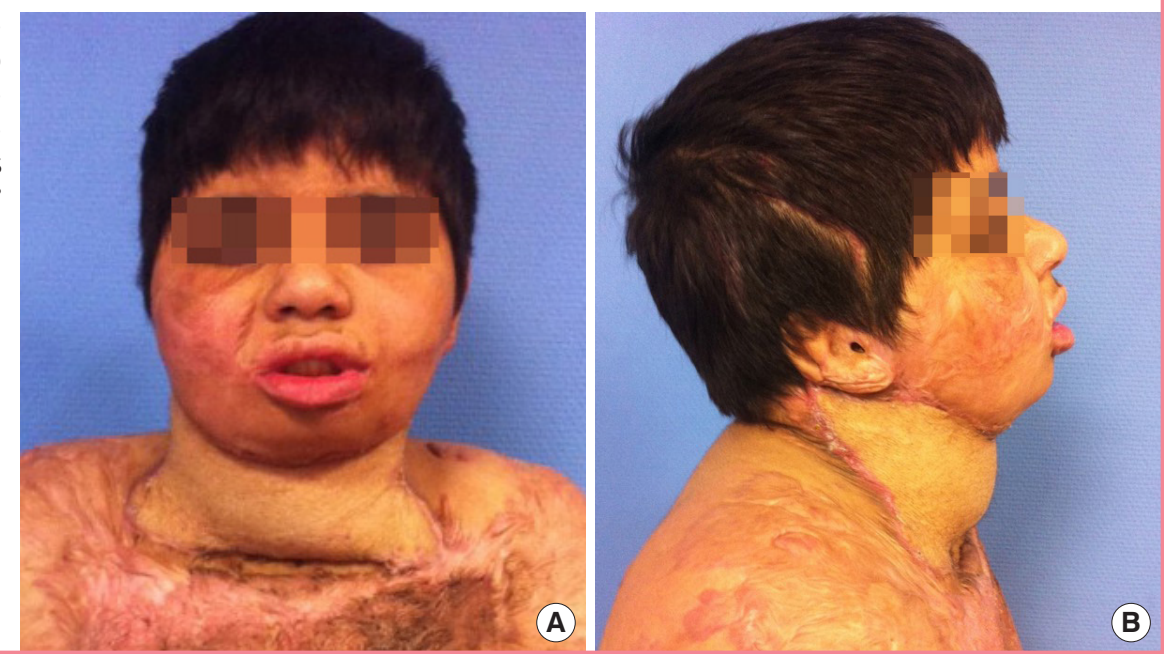

Fig. 5. Case 2

A 23-year-old woman patient: (A) at 4 years' follow-up following butterfly anterolateral thigh flap resurfacing and additional liposuction (at postoperative 1 year), with marked improvement in aesthetics of the neckline and (B) improvement in neck extension (from $90^{\circ}$ to $\left.120^{\circ}\right)$.
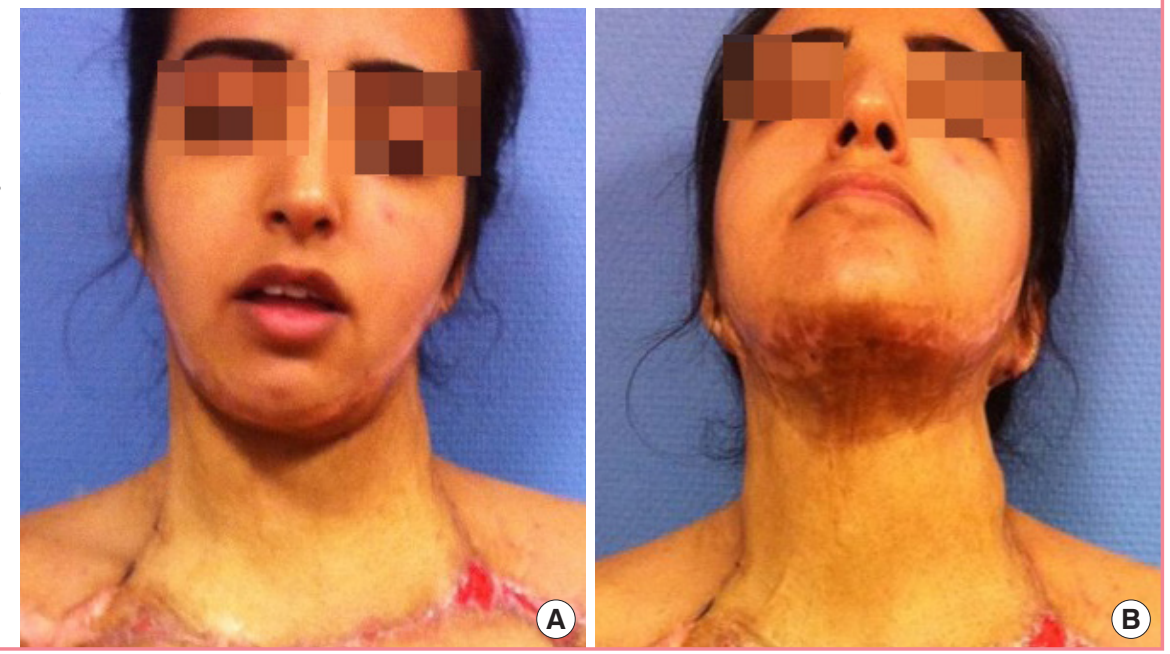

rior neck is involved, loco-regional options become severely limited; STSGs are also far from ideal due to their inherent poor cosmetic result, in addition to the need for prolonged immobilization and pressure dressings [9]. Furthermore, Asian patients tend towards a greater inflammatory response to wounding and therefore, are at greater risk for developing burn contractures [10].

Free tissue transfers provide full-thickness skin for superior resurfacing and have previously been demonstrated to be reliable and safe for reconstruction of head and neck burns, with a technical success rate of $94 \%$ [8]. However, this is not without its drawbacks such as the need for a prolonged, complex procedure and the near obligatory requirement for future debulking procedures (up to $64 \%$ incidence) [8]. With improved anatomical knowledge, thinner, wider and larger flaps such as the free fascio-cutaneous ALT flap can now be utilized to address the chal- lenges of reconstructing anterior neck burn contractures. Various modifications to further optimize the use of the ALT for this purpose include pre-expansion to thin out the flap further [11] and prefabrication at distant sites to obtain a thinner flap [8]. These procedures are all staged however, and are not without risks of infection and pedicle thrombosis respectively.

In proposing our butterfly design for the ALT flap, we hope to provide a simple modification to further improve on the aforementioned advancements. By matching the dimensions of the skin defect better following scar excision, this would enable wound closure to have less tension as compared to the traditional, elliptical design of the ALT flap during insetting. In turn, this may help maintain flap mobility and that of the reconstructed neck at the cervico-mental angle, thereby further reducing the risk of developing scar contractures. Additionally, the butterfly design of the ALT flap renders the donor site at the thigh 
amenable to closure in V-Y fashion, which would arguably reduce the risks of further donor site morbidity such as dehiscence and/or need for additional skin grafting.

In both cases, platysmaplasty was not necessary as the muscle was not involved nor scarred. There were no local complications such as flap losses or donor site dehiscence. Length of hospital stay was 7 and 5 days respectively without the need for extra rehabilitation. Neck extension improved by $37^{\circ}$ and $30^{\circ}$ to normal $\left(>110^{\circ}\right)$ at 4 years' follow-up with no evidence of contracture recurrence. While further debulking was required in both patients, this is a trade-off that can be performed on an outpatient basis and the risks of which would certainly be better tolerated compared to the need for multiple staged procedures. Ultimately, we believe that this butterfly design of the ALT flap is valuable for both primary and secondary reconstruction of anterior neck burns and affords all the advantages of free tissue transfers without the inherent risks of multiple staged procedures.

\section{NOTES}

\section{Conflict of interest}

No potential conflict of interest relevant to this article was reported.

\section{Ethical approval}

The study was performed in accordance with the principles of the Declaration of Helsinki.

\section{Patient consent}

The patients provided written informed consent for the publication and the use of their images.

\section{Author contribution}

Study design: Lantieri LA. Data collection: Lellouch AG, Pozzo V, Suffee T. Manuscript writing and critical review: Lellouch AG, Ng ZY, Lantieri LA. Review of the manuscript: Pozzo V, Suffee T.

\section{ORICD}

Alexandre G. Lellouch

https://orcid.org/0000-0001-8191-8662

Zhi Yang Ng https://orcid.org/0000-0003-0538-3066

\section{REFERENCES}

1. Koerhuis CL, Winters JC, van der Helm FC, et al. Neck mobility measurement by means of the 'Flock of Birds' electromagnetic tracking system. Clin Biomech (Bristol, Avon) 2003;18:14-8.

2. Hamilton TJ, Patterson J, Williams RY, et al. Management of head and neck burns: a 15-year review. J Oral Maxillofac Surg 2018;76:375-9.

3. Gandolfi S, Carloni R, Bertheuil N, et al. Assessment of quality-of-life in patients with face-and-neck burns: the Burn-Specific Health Scale for Face and Neck (BSHS-FN). Burns 2018;44:1602-9.

4. Pribaz JJ, Caterson EJ. Evolution and limitations of conventional autologous reconstruction of the head and neck. J Craniofac Surg 2013;24:99-107.

5. Yang JY, Tsai FC, Chana JS, et al. Use of free thin anterolateral thigh flaps combined with cervicoplasty for reconstruction of postburn anterior cervical contractures. Plast Reconstr Surg 2002;110:39-46.

6. Makboul M, El-Oteify M. Classification of post-burn contracture neck. Indian J Burn 2013;21:50-4.

7. Luo X, Liu F, Wang X, et al. Region-oriented and staged treatment strategy in reconstruction of severe cervical contracture. PLoS One 2015;10:e0122669.

8. Parrett BM, Pomahac B, Orgill DP, et al. The role of free-tissue transfer for head and neck burn reconstruction. Plast Reconstr Surg 2007;120:1871-8.

9. Achauer BM. Reconstructing the burned face. Clin Plast Surg 1992;19:623-36.

10. Noda Y, Kuwahara H, Morimoto M, et al. Reconstruction of anterior neck scar contracture using a perforator-supercharged transposition flap. Plast Reconstr Surg Glob Open 2018;6:e1485.

11. Acarturk TO. Aesthetic reconstruction of the postburn neck contracture with a preexpanded anterolateral thigh free flap. J Craniofac Surg 2014;25:e23-6. 